\title{
Infección pulmonar por Mycobacterium kansasii y carcinoma broncopulmonar sincrónico
}

\author{
J. SANCHO CHINESTA, F. CARRIÓN VALERO \\ Servicio de Neumología. Hospital Clínico Universitario. Universitat de València
}

\author{
LUNG INFECTION FOR MYCOBACTERIUM KANSASII AND \\ CARCINOMA OF THE LUNG SYNCHRONOUS
}

\begin{abstract}
RESUMEN
Aunque la asociación de tuberculosis pulmonar y carcinoma de pulmón es bien conocida en la literatura, resulta excepcional la presentación sincrónica de la infección pulmonar por micobacterias atípicas y el carcinoma broncopulmonar. Por ello, presentamos el caso de un varón de 41 años, fumador, con antecedentes de tuberculosis pulmonar en la adolescencia y hepatopatía crónica por virus $\mathrm{B}$ y $\mathrm{C}$, que acudió a nuestra consulta externa por presentar febrícula, disfonía y pérdida de $4 \mathrm{~kg}$ de peso de un mes de evolución. La TC de tórax reveló una masa apical izquierda con extensión a la pared y adenopatías mediastínicas, así como un infiltrado pulmonar en LSI. La biopsia de una adenopatía supraclavicular mostró metástasis de carcinoma epidermoide, mientras que en el esputo se aisló Mycobacterium kansasii sensible a rifampicina y etambutol. La serología VIH fue negativa.

Se comentan los posibles mecanismos de esta infrecuente asociación, junto a una revisión de la literatura.
\end{abstract}

PALABRAS CLAVE: Carcinoma broncopulmonar. Mycobacterium kansasii.

\begin{abstract}
The association of lung tuberculosis and carcinoma of the lung is very well-known, although it is exceptional the synchronous presentation of lung infection for nontuberculous mycobacteria and lung carcinoma. We present the case of a 41 yearold male, smoker, with antecedents of lung tuberculosis in the adolescence and chronic liver disease for virus $B$ and $C$ that he presents fever, hoarseness and loss of $4 \mathrm{~kg}$ of weight of a month of evolution. The CT scan of the chest revealed a lung mass in left

apex with pneumonitis, tumor invading chest wall and mediastinal lymph node enlargement. The biopsy of a supraclavicular node showed metastasisof squamous carcinoma, while in the sputum was isolated Mycobacterium kansasii seneitive to rifampin and ethambutol. HIV was negative.

The possible mechanisms of this uncommon association are com mented, next to a revision of the literature.
\end{abstract}

KEY WORDS: Carcinoma of the lung. Mycobacterium kansasii.

Sancho Chinesta J, Carrión Valero F. Infección pulmonar por Mycobacterium kansasii y carcinoma broncopulmonar sincrónico. An Med Interna (Madrid) 2002; 19: 186-188.

\section{INTRODUCCIÓN}

La asociación de tuberculosis pulmonar (TBC) y neoplasias de pulmón es un hecho conocido desde hace años. Se puede desarrollar un carcinoma broncogénico sobre procesos cicatriciales secundarios a TBC o bien aparecer sincrónicamente ambos (1). La presentación simultánea de ambos procesos es poco frecuente, 1 a $3 \%$ de los pacientes con cáncer de pulmón según las series (2).

Presentamos un caso de infección pulmonar activa por Myco bacterium kansasii sincrónica con carcinoma broncogénico.

\section{CASO APORTADO}

Paciente varón de 41 años fumador de 20 cigarrillos al día con antecedentes de hepatitis por VHC y VHB y TBC pulmonar en la adoles- cencia con lesiones residuales fibrosas en vértice pulmonar izquierdo. Ingresa por presentar un cuadro clínico de un mes de evolución caracterizado por febrícula de predominio vespertino, sudoración profusa, pérdida de $4 \mathrm{~kg}$ de peso y disfonía. A la exploración destaca roncus aislados a la auscultación pulmonar y adenopatía supraclavicular izquierda no dolorosa fija y adenopatías axilares móviles, blandas y no dolorosas. La radiografía de tórax muestra infiltrado cavitado en lóbulo superior izquierdo con pérdida de volumen y engrosamiento pleural apical izquierdo. En la analítica destaca 13170 leucocitos con fórmula normal, GOT 89 UI/I, GPT 102 Ul/l y LDH 681 UI/I. Mantoux de 9 mm de induración. En el esputo se aislaron BAAR siendo el cultivo positivo tanto en medio Bactec como en Lowestein-Jensen para Mycobacterium kansasii, sensible a rifampicina y etambutol. La exploración ORL informa como parálisis recurrencial de cuerda vocal izquierda. La TAC toracocervical muestra masa apical izquierda de $4 \mathrm{~cm}$ con afectación de pared, engrosamiento pleural apical izquierdo, adenopatías prevasculares, en ventana aortopulmonar y región supraclavicular izquierda; condensación con broncograma aéreo en segmento posterior del lóbulo

Trabajo aceptado: 14 de septiembre de 2000

Correspondencia: Francisco Carrión Valero. Servicio de Neumología. Hospital Clínico Universitario Avda. Blasco Ibáñez, 17. 46010 Valencia 


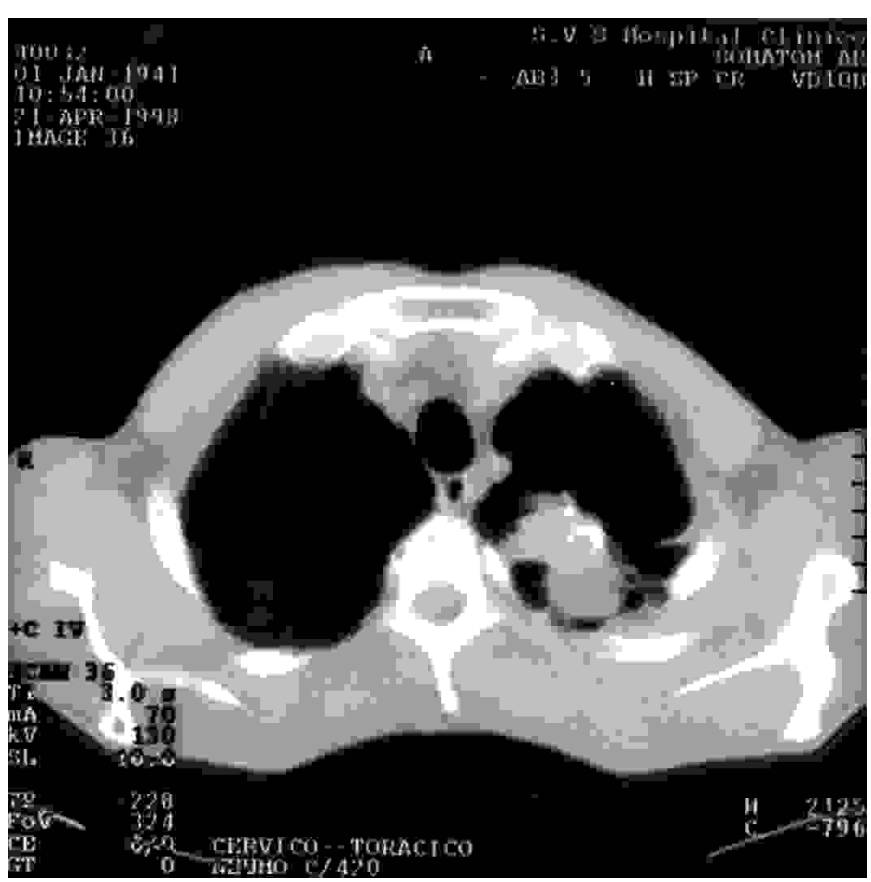

Fig. 1. TC tórax que muestra infiltrado pulmonar y masa apical izquierda con extensión a la pared torácica.

superior izquierdo. La fibrobroncoscopia muestra mucosa hiperémica del BLSI siendo las citologías negativas. La biopsia de la adenopatía supraclavicular izquierda se informa como metástasis por carcinoma epidermoide escasamente diferenciado. Se inicia tratamiento con isoniacida, rifampicina y etambutol y radioterapia.

\section{DISCUSIÓN}

La asociación de TBC y enfermedades neoplásicas es un hecho constatado por múltiples autores (3), fundamentalmente en lo que se refiere a enfermedad de Hodgkin, leucemia linfática crónica y neoplasias de las vías aéreas y del tracto digestivo superior (4). A nivel pulmonar parece ser que la incidencia de carcinoma broncogénico es superior en los pacientes con TBC que en la población general; esto podría explicarse por varios hechos. En primer lugar la predisposición a desarrollar neoplasias, sobre todo adenocarcinomas, en zonas cicatriciales parenquimatosas (5); por otro lado la TBC puede reactivarse tras la erosión de los focos caseosos encapsulados, por la infiltración de células neoplásicas (6); la situación de inmunosupresión por alteración de la inmunidad celular generada ya sea por la propia neoplasia o por los tratamientos recibidos, quimio y radioterapia, o por la caquexia y malnutrición que conllevan las enfermedades malignas, que favorecerían la infección o la reactivación de la TBC (7).

En el caso que presentamos, la presencia de carcinoma epidermoide metastásico junto con imágenes cavitadas en la radiografía de tórax y aislamiento de bacilos ácido-alcohol resistentes en el esputo nos hizo sospechar inicialmente reactivación de TBC antigua en paciente con carcinoma broncogénico, si bien la identificación de Mycobacterium kansasii a partir de los cultivos nos obligó a replantearnos la situación.
Mycobacterium kansasii es una micobacteria fotocromógena ya que en medios de cultivo adecuados puede producir colonias no pigmentadas en la oscuridad que si se exponen a la luz adquieren una coloración amarillo-anaranjada debido al depósito de cristales de betacaroteno. A diferencia de $M y c o$ bacterium tuberculosis no produce niacina. Son micobacterias de crecimiento lento cuyo reservorio principal es el medio ambiente (8). Sólo se ha descrito un serotipo.

En nuestro medio Mycobacterium kansasii representa la causa más frecuente de afectación pulmonar por micobacterias no tuberculosas (9). Afecta a pacientes de mayor edad que Mycobacterium tuberculosis con enfermedades sistémicas o pulmonares crónicas de base, sobre todo alcohólicos, enfermedad pulmonar obstructiva crónica o neumoconiosis entre otras, siendo capaz de producir enfermedad diseminada en pacientes con alteración de la inmunidad celular como los tratados con glucocorticoides e inmunosupresores, trasplantados, enfermedades autoinmunes y hematológicas y en pacientes con síndrome de inmunodeficiencia adquirida (10). Asimismo puede haber afectación cutánea por inoculación local tanto en inmunocompetentes como en inmunodeprimidos.

Se han descrito casos de infección pulmonar por Myco bacterium kansasii y carcinoma broncogénico sincrónico; Lillo y cols. (11) describen que de 44 pacientes en donde se aisló Mycobacterium kansasii, 6 presentaban carcinoma broncogénico; Echevarria y cols. (12) encontraron que el 7,4\% de los pacientes con infección por Mycobacterium kan sasii presentaban una neoplasia, sin especificar el origen. Las causas por las que aparecerían ambos procesos sincrónicamente serían las mismas que las descritas para la TBC pulmonar (13). La alteración del aclaramiento mucociliar y de la función de los macrófagos se considera que juegan un papel primordial en la patogénesis de la infección (14).

La infección por Mycobacterium kansasii se adquiere a partir del medio, a través de agua contaminada o de aerosoles, siendo rara la transmisión interhumana aunque se han descrito casos de afectación familiar (14). Mycobacterium kansasii raramente coloniza; Lillo (11) observó una proporción de enfermedad/colonización de 10/1 por lo que su aislamiento en número significativo de colonias es sinónimo de enfermedad.

Para poder diferenciar colonización, contaminación y enfermedad se siguen los criterios de la American Thoracic Society (16): cuadro clínico radiográfico compatible con TBC pulmonar activa; aislamiento por cultivo de múltiples colonias de la misma especie de micobacterias en dos o más esputos o en esputo y BAS; exclusión de otras posibles causas de enfermedad. Si bien en pacientes inmunodeprimidos, sobre todo en relación con la infección por VIH muchos autores cuestionan tales criterios.

Clínicamente la infección pulmonar es insidiosa, de características similares a la producida por Mycobacterium tuber culosis con fiebre, tos productiva, pérdida de peso. A nivel radiográfico las imágenes pueden ser muy variadas, si bien existe una gran tendencia a las lesiones cavitarias de predominio en lóbulos superiores (17) que se observan entre el 43 y el $96 \%$ de los casos según las series (18).

La enfermedad diseminada se produce por vía hematógena a partir de un foco pulmonar, caracterizándose por fiebre, astenia, pérdida de peso, adenopatías diseminadas y clínica de órgano afecto. La afectación cutánea puede incluir nódulos, pústulas, verrugas, placas, abscesos, seromas y úlceras. 
La combinación de isoniacida (300 mg/día), rifampicina (600 mg/día) y etambutol $(25 \mathrm{mg} / \mathrm{kg} /$ día los dos primeros meses y luego $15 \mathrm{mg} / \mathrm{kg}$ ) suele ser el tratamiento inicial de elección en una pauta de 18 o de 12 meses (19). La rifampicina se considera el fármaco más eficaz, siendo sólo el 15 a $30 \%$ de las cepas sensibles a isoniacina en concentraciones altas; la pirazinamida no es efectiva trente a Mycobacterium kansasii, habiéndose encontrado resistencias in vitro frente a ella.

\section{Bibliografía}

1. Fernandez de Sevilla T, Alegre J, Fernández A, Pigram C, Martín Vazquez JM. Adenocarcinoma y tuberculosis pulmonar activa. Rev Clin Esp 1988; 192: 393.

2. Martínez E, Aparicio J, Cordero P, Sanchis J, Leon M, Perpiñá M. Coexistencia de carcinoma broncogénico y tuberculosis pulmonar activa. Arch Bronconeumol 1995; 31: 32-34.

3. Komatsu H, Nagai H, Kurashima A, Shishido H, Machida K, Kawabe Y, Akagava S, Ootsuka Y, Naguyama N. Association of active pulmonary tuberculosis and malignant diseases: a clinical study. Kekkaaku 1995; 70: 281-284.

4. Kaplan MH, Armostron D, Rosen P. Tuberculosis complicating neoplastic disease.A review of 201 cases. Cancer 1974; 33: 850-858.

5. Garrapiz J, Serrano R, Aznar R. Carcinoma bronquioloalveolar y tuberculosis pulmonar activa. Arch Bronconeumol 1990; 26: 378.

6. Tomas S, Pons A, Planas J, Gallen M, Malats N, Botet J. Diagnóstico sincrónico de cáncer de pulmón y tuberculosis pulmonar activa. Neoplasia 1990; 6: 14-16.

7. Feld R, Bodey GP, Groschel D. Mycobacteriosis in patients vith malignant disease. Arch Inter Med 1976; 136 67-70.

8. Ruiz Manzano J, Manterola JM, Ausina V, Sauret J.Nomenclatura y clasificación de las Micobacterias.Arch Bronconeumol 1998; 34: 154-157.

9. Hernández S, Sauret J, Ausina V et al.Enfermedad pulmonar por micobacterias ambientales oportunistas. Revisión de 35 casos. Med Clin (Barc) 1990; 95: 53-56.

10. Castro J, Sánchez JM, Fernández a Antón J. Infección diseminada
En caso de resistencia a la rifampicina o fracaso del tratamiento se deben utilizar tres fármacos no utilizados anteriormente, considerándose como altemativas la isoniacida a dosis altas (600 mg), el sultametoxazol, la claritromicina y las fluorquinolonas

Para la afectación extrapulmonar se debe de utilizar el mismo régimen que el propuesto para la enfermedad pulmonar (16).

por Mycobacterium Kansasii con afectación cutánea en una paciente con síndrome de inmunodeficiencia adquirida. Med Clin (Barc) 1998; 110: 598.

11. Lillo M, Orengo S, Cernoch P, Harris RL. Pulmonary and disseminated infection due to Mycobacterium Kansasii: a decade of experience. Rev Infect Dis 1990; 12: 760-767.

12. Echevarría MP, Martin G, Pérez J, Urkijo JC. Enfermedad pulmonar por Mycobacterium Kansasii.Presentación de 27 casos (1988-1992). Enfer Infec Microbiol Clin 1994; 12: 280-284.

13. Zvetina JR, Maliwan N. Mycobacterium Kansasii infection following Primary Pulmonary Malignancy.Chest 1994; 105: 325-326.

14. Zvetina JR, Maliwan N, Frederick WE, Reyes C. Mycobacterium Kansasii infection following Primary Pulmonary Malignancy. Chest 1992; 102: 1460-1463.

15. Woodring JH, Vandiviere H. Pulmonary disease caused by nontuberculous mycobacteria. J Thrac Imag 1990; 5: 64-76.

16. American Thoracic Society. Diagnosis and treatment of Nontuberculous Mycobacteria.Am Rev Respir Dis 1990; 142: 940-953.

17. Gallont JE, Ko AH. Cavitary pulmonary lesions in patients infected with human inmunodeficiency virus.Clin Infect Dis 1996; 22: 671-682.

18. León M, Sánchez P, De Diego A, Otero E.lnfección pulmonar por Mycobacterium Kansasii tras neumonía por citomegalovirus en paciente con trasplante renal. Arch Bronconeumol 1994; 30: 222-225.

19. Sauret J, Hernández Flix S, Castro E.Treatment of pulmonary disease caused by Mycobacterium Kansasii. Results of 18 v.s. 12 months chemotherapy.Tubercle Lung Dis 1995; 76: 104-108. 